\title{
Interacción natural a través un chatbot de consejería de salud sexual y reproductiva dirigida a jóvenes universitarios
}

\author{
Natural interaction through a sexual and reproductive health advice \\ chatbot, aimed at young university
}

Recibido: noviembre 18 de 2021 | Revisado: noviembre 24 de 2021 | Aceptado: diciembre 02 de 2021

\author{
Elizabeth Valladares SÁNCheZ \\ Miguel Estrella Santillán ${ }^{\mathrm{I}}$ \\ Norma LeÓn Lescano ${ }^{\mathrm{I}}$
}

\begin{abstract}
Resumen
El objetivo de este trabajo es describir la implementación de una solución de procesamiento de lenguaje natural conversacional en un chatbot llamado TELENANU para realizar consejería sexual reproductiva y validar su impacto en jóvenesuniversitarios. El método aplicado contempla cuatro etapas iterativas: definir lo que se quiere del software, construir el software que incluye la técnica de implementación de inteligencia artificial, realizar las pruebas y aprender, documentar lo aprendido y difundir conocimiento. La solución recibió una buena aceptación por parte del público objetivo. Se logró difundir el servicio en tres redes sociales Facebook, Instagram y TikTok y en tres canales: página web, Microsoft Teams y Telegram.
\end{abstract}

Palabras clave: Chatbot; consejería sexual y reproductiva; jóvenes universitarios; procesamiento de lenguaje natural

\begin{abstract}
The objective of this work is to describe the implementation of a conversational natural language processing solution in a chatbot called Telenanu, to perform reproductive sexual counseling and validate its impact on young university students. The applied method includes four iterative stages, defining what is wanted from the software, building the software that includes the artificial intelligence implementation technique, conducting tests and learning, documenting what has been learned and disseminating knowledge. The solution was well accepted by the target audience, the service was spread on three social networks: Facebook, Instagram and TikTok; and in three channels, website, Microsoft Teams, Telegram.
\end{abstract}

Key words: Chatbot; sexual and reproductive counseling; young university students; natural language processing
Autor para correspondencia

E-mail: nleonl@usmp.pe
1 Universidad de San Martín de Porres, Lima, Perú

Correo: elizabeth_lopez1@usmp.pe

(C) Los autores. Este artículo es publicado por la Revista Campus de la Facultad de Ingeniería y Arquitectura de la Universidad de San Martín de Porres. Este artículo se distribuye en los términos de la Licencia Creative Commons Atribución No-comercial - Compartir-Igual 4.0 Internacional (https://creativecommons.org/licenses/ CC-BY), que permite el uso no comercial, distribución y reproducción en cualquier medio siempre que la obra original sea debidamente citada. Para uso comercial contactar a: revistacampus@usmp.pe.

https: 


\section{Introducción}

La educación sexual en los países de desarrollo ha alcanzado cierto grado de aceptación al tratar la higiene personal y los cuidados preventivos; sin embargo, a través de la historia ha sido considerado tabú debido a las tradiciones y creencias (García, 2007). Asimismo, los jóvenes tienden a sentir la presión social debido a como se conceptualiza el tema de salud sexual en la sociedad, ligándolo a los actos sexuales, los riesgos que puede tener su práctica y a las doctrinas religiosas (Sanjuán, 2020).

En el Perú, las relaciones sexuales a temprana edad son muy comunes, según las cifras de las encuestas demográficas y de salud familiar, donde alrededor del 64\% de mujeres menores de 19 años tuvo su primera relación sexual y el 35.6\% entre los 20 a 24 años. En el caso de Lima Metropolitana, el 66.3\% de mujeres menores de 19 años y el 33.2\% entre los 20 a 24 años (ENDES, 2019).

Las féminas, sexualmente activas entre los 15 a 24 años, se embarazan a una temprana edad en un $22.6 \%$ lo que origina la deserción en sus estudios. Además, se obtuvo que en Lima Metropolitana el $25 \%$ de las féminas entre los 15 a 24 años usa métodos anticonceptivos, el 14.9\% usa algún método tradicional y el $60.1 \%$ algún método moderno (INEI - Perú: Encuesta Demográfica y de Salud Familiar 2019 - Nacional y Regional, 2019).

El estado de emergencia por Covid-19 impactó en todos los sectores privados y públicos del Perú. Se enfrentó un cambio forzoso hacia la transformación digital adaptando servicios y productos que afectan directamente los servicios sanitarios de emergencia (Carbajo Martín, 2020). La salud sexual quedó rezagada a segundo plano, dejando descubierta la demanda de los servicios de planificación familiar. Los horarios de atención para el servicio de salud sexual y reproductiva se limitaron entre las 8 a.m. y el mediodía. Además, se disminuyó la atención rutinaria para el uso de anticonceptivos, la salud reproductiva, la atención prenatal, entre otros (Camacho et al., 2020).

El estado peruano cuenta con planes estratégicos para atender las necesidades de las etapas de vida del ser humano a través de la cobertura médica; sin embargo, los jóvenes al cumplir la mayoría de edad pierden estos derechos, lo cual implica que tengan que asumir dichos costos. Este grupo de jóvenes se ve obligado a adquirir un seguro médico, pero debido a que son económicamente dependientes y a la escasa oportunidad de trabajo, la vulnerabilidad de este sector poblacional aumenta y son candidatos a presentar inestabilidad en su salud sexual reproductiva. Como consecuencia de esta situación, buscan otros medios de atención $\mathrm{u}$ orientación como el internet sin tener en cuenta la fiabilidad de la información y exponiéndose a riesgos de salud sexual y reproductiva (Acurio Gamarra \& Vergara Rodríguez, 2020).

En respuesta a la situación comentada, el Centro Especializado en Salud Reproductiva del Adolescente (CESRA) de la facultad de Obstetricia y Enfermería en conjunto con el área de Laboratorio de Software y Tecnologías Interactivas (LABSTI) de la facultad de Ingeniería y Arquitectura de la Universidad de San Martín de Porres presentaron un proyecto llamado Telenanu, el cual es un agente 
conversacional que brinda el servicio de consejería virtual en salud sexual y reproductiva orientado a los adolescentes y jóvenes que cumplan el requisito de mayoría de edad, en respuesta al cese de la atención de salud sexual y reproductiva presencial por parte del CESRA a causa de la pandemia Covid-19 (Justiniano, 2021).

Sin embargo, el chatbot evidenció problemas de interacción natural con los jóvenes. Por tanto, el objetivo del proyecto fue implementar nuevos escenarios del flujo de la conversación entre el usuario y el chatbot, además, de considerar la diversificación de canales de comunicación ya que el chatbot brindaba sus servicios en página web.

$\mathrm{Al}$ analizar los problemas, se planteó la siguiente pregunta ¿Cómo construir e integrar interacción natural entre el chatbot conversacional Telenanu y los individuos jóvenes, en el contexto de una consejería de salud sexual y reproductiva? Al buscar responder esta pregunta, la investigación entró en un proceso de prueba, aprendizaje y documentación de lo obtenido. En síntesis, el presente artículo relata y explica el desarrollo de un chatbot capaz de realizar consejería sexual reproductiva y validar su impacto en jóvenes universitarios.

Para alcanzar el objetivo trazado, se planteó la integración del chatbot conversacional a las redes sociales más populares entre los jóvenes, integrar los canales de comunicación del chatbot conversacional e implementar inteligencia artificial al chatbot conversacional para comprender y entregar respuestas a la solicitud de consultas de salud sexual y reproductiva.

\section{Método}

El método incluyó una investigación preliminar sobre soluciones con chatbot a problemas surgidos en la pandemia. Se encontró que en el ańo 2020, en España, se realizó un estudio sobre los chatbot como solución de comunicación durante la crisis por el Covid-19. La investigación concluye que los agentes conversacionales mejoran la experiencia de usuarios, y, el atractivo principal de estos está en la atención múltiple y el servicio de 24/7 a la semana, además de las respuestas rápidas. Otro factor clave son las plataformas de medios sociales como Facebook, Instagram, WhatsApp y Twitter como principales medios para brindar información (Bonales et al., 2021).

Catalina es un chatbot ecuatoriano que se encarga de atender consultas referentes al Covid -19 e informar a los ciudadanos. Posee 79 canales mediante páginas web y WhatsApp, por lo que tiene un gran alcance en la interacción con los usuarios (Torre Juana, 2020).

La Universidad de Alicante desarrolló un Chatbot para responder preguntas con relación a sus servicios. El estudio determinó que el canal determinante sería el sitio web de la institución y el uso del Messenger de Facebook por ser el medio más usado entre las redes sociales (Valero Clavel, 2019). Finalmente, se puso atención en una investigación que implementó un chatbot para predecir y prevenir enfermedades mentales en una fase temprana del ser humano, con tecnología NPL para el análisis de datos, en donde el usuario al ingresar los datos estos pasan por un preprocesamiento, para el cual se mencionan dos técnicas: 
La eliminación de ruido y mayúscula/ minúscula, la primera consiste en eliminar caracteres superfluos y la segunda consiste en transformar todas las letras que se encuentren de mayúscula a minúsculas (Podrazhanshy et al., 2020).

Después de esta investigación, se logró definir el marco de trabajo, el cual tuvo cinco etapas iterativas: (1) Definir lo que se quiere del software, (2) Construir el software, (3) Realizar pruebas y aprender de ello, (4) Documentar lo aprendido y difundir conocimiento. El proceso se repite una y otra vez a medida en que se avance mejoras o nuevas características en el software, ¡Error! No se encuentra el origen de la referencia..

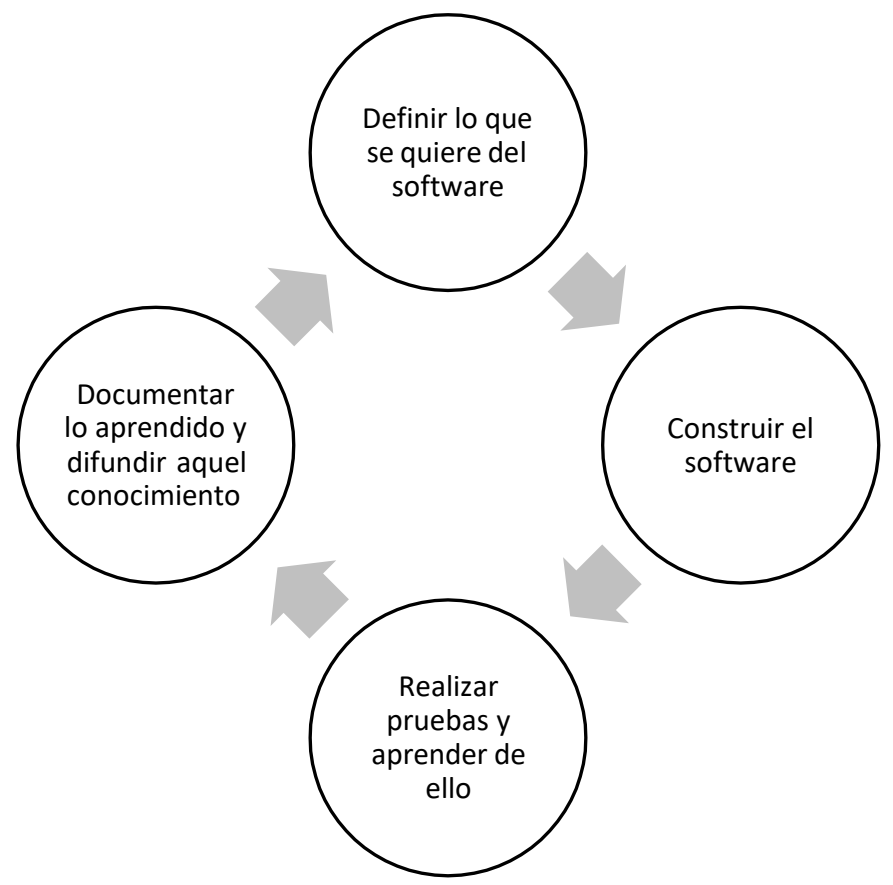

Figura 1. Etapas de desarrollo metodológico del chatbot

Nota. Shevat, 2017

En la primera etapa, definir lo que se quiere del software consta de dos procesos: definición y recopilación de requerimientos, y el análisis del flujo conversacional. En el primer proceso, se realizó la recopilación de la información y desarrolló la técnica Wizard of Oz. Para ello, se contó con el trabajo de tres investigadoras quienes a través de la aplicación de mensajería Facebook Messenger se hicieron pasar por el chatbot TELENANU para brindar el servicio de consejería virtual en salud sexual y reproductiva a razón de evaluar las conductas del usuario con el chatbot y determinar la personalidad de este, el cual se determinó por una personalidad amigable y cordial. En el segundo proceso, denominado Flujo conversacional, se realizó una recopilación de información solicitada a las investigadoras especialistas obstetras en salud sexual y reproductiva (SSR) donde se tratan los temas de: Infecciones sexuales, cambios corporales, ayuda por violencia sexual, ayuda por posibilidad de embarazo, cáncer ginecológico, inicio de relaciones sexuales y métodos anticonceptivos; Figura 2, una 
vez recopilada la información, se realizó el análisis del guion, que presentara el chatbot para generar la interacción con los usuarios dando como resultado un flujo conversacional; además, se analizó el uso de los canales con los cuales el Bot debería contar.

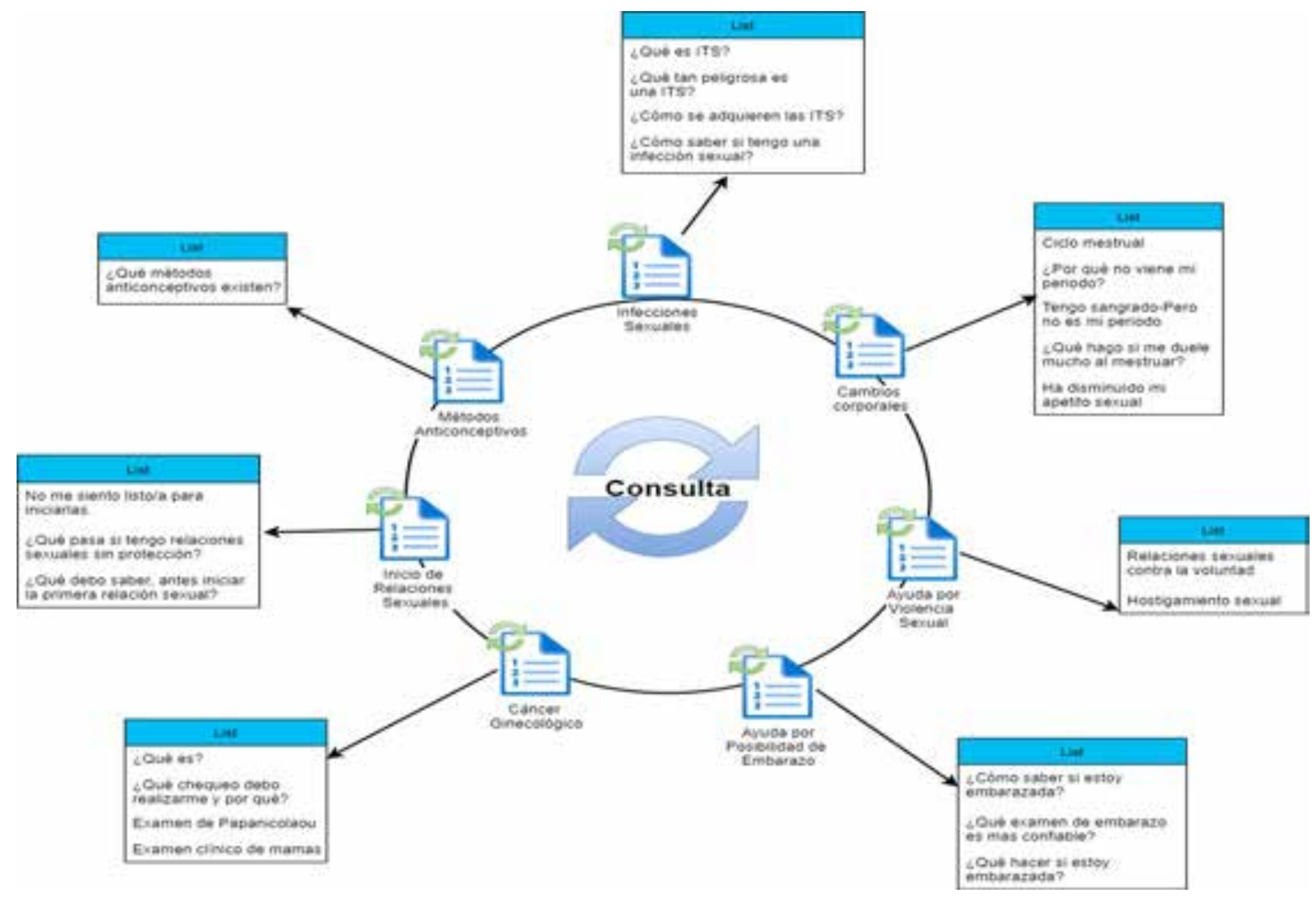

Figura 2. Información recopilada de la consulta de SSR

En la segunda etapa, Construir el software, se realizaron tres iteraciones. En la primera iteración, se ejecutó el diseño y prueba de herramientas. Se efectuó la evaluación y prueba del entorno de desarrollo más viable y eligió la plataforma de Azure Bot Service debido a la capacidad de cumplir con las normas HIPAA para el tratamiento de la información relacionada al ámbito de la salud.

A continuación, se diseñó la implementación de la inteligencia artificial al chatbot para permitir una conversación natural durante la consejería y con ello ganar la confianza del estudiante.

En la tercera iteración, se creó el Chatbot, para lo cual se planteó el desarrollo de la técnica de implementación de software con NLP dividido en seis procesos: (1) Recolección de datos, que refiere a la etapa del flujo conversacional; (2) Preprocesamiento de datos, que hace uso de la herramienta "Language Understanding" (LUIS) de Microsoft Azure en donde se usó el aprendizaje automático para predecir las intenciones de los estudiantes además de extraer nueva información; (3) Ingeniería de características, este busca analizar la palabra clave dentro de los datos de entrenamiento ingresados a LUIS para generar un cálculo decimal a razón de identificar la intención del usuario; (4) Desarrollo de modelos, desarrollo de los escenarios en Azure Healt Bot donde se irá actualizando y mejorando iterativamente con la recopilación de nueva información; 
(5) Evaluación de modelos, con el uso de LUIS se realiza la operación Test donde se mostrará los resultados sobre cuál es la intención según un porcentaje de aproximación y la entidad según el tipo; y la (6) Implementación, despliegue y supervisión se desarrolla en la etapa final donde se generan reportes de las consultas realizadas al chatbot y el análisis de anomalías en el rendimiento del chatbot con el uso de Azure Insight.

En la tercera etapa, Realizar pruebas y aprender de ello, constó de un ciclo de pruebas de comunicación, en paralelo a la etapa de construcción. Con un grupo de usuarios se realizaban las pruebas, documentaban los resultados, corregía, aprendía y continuaba el ciclo iterativo.

La cuarta, era documentar lo aprendido y difundir conocimiento. Consta de la realización de la analítica y mejora continua la cual busca la conversión de los datos no estructurados sin procesar a información estructurada procesable para obtener una mejor visualización de los datos por medio de paneles que permitan el análisis de la información, realizar ajustes y difundir los resultados.

\section{Resultados y Discusión}

Para validar el impacto de la consejería sexual reproductiva, realizada por TELENANU a jóvenes universitarios, se utilizó el análisis de correlación para encontrar el grado intuitivo de la aplicación, interacción, respuestas validas, y el nivel de recomendación de los jóvenes para TELENANU.

A través del análisis de correlación de Spearman, se analizó la variable de sexo y la página web intuitiva que dio por resultado 0.267 según se observa en la Tabla 1, por lo cual es aceptada. Esto significa que la página web es intuitiva tanto para varones como para mujeres.

Tabla 1

Correlación entre el sexo y la UX

\begin{tabular}{|c|c|c|c|c|}
\hline \multirow{7}{*}{ Rho de Spearman } & \multicolumn{3}{|r|}{ Sexo } & UX \\
\hline & Sexo & Coeficiente de correlación & 1,000 & 0,267 \\
\hline & & Sig.(bilateral) & & 0,153 \\
\hline & & $\mathrm{N}$ : muestra & 30 & 30 \\
\hline & UX & Coeficiente de correlación & 0,267 & 1,000 \\
\hline & & Sig.(bilateral) & 0,153 & \\
\hline & & N: muestra & 30 & 30 \\
\hline
\end{tabular}

Respecto al inicio de la conversación con el chatbot, se analizaron la variable sexo y la variable de inicio de la conversación aceptable que dio por resultado 0.148 según la Tabla 2 por lo cual es aceptado; es decir, que el inicio de la conversación con el chatbot les parece aceptable tanto para varones como mujeres. 
Tabla 2

Correlación entre el sexo y el inicio del Chat

\begin{tabular}{|c|c|c|c|c|}
\hline \multirow{7}{*}{$\begin{array}{l}\text { Rho de } \\
\text { Spearman }\end{array}$} & & & Sexo & Inicio Chat \\
\hline & \multirow[t]{3}{*}{ Sexo } & Coeficiente de correlación & 1,000 & 0,148 \\
\hline & & Sig.(bilateral) & & 0,434 \\
\hline & & $\mathrm{N}$ : muestra & 30 & 30 \\
\hline & \multirow[t]{3}{*}{ Inicio Chat } & Coeficiente de correlación & 0,148 & 1,000 \\
\hline & & Sig.(bilateral) & 0,434 & \\
\hline & & $\mathrm{N}$ : muestra & 30 & 30 \\
\hline
\end{tabular}

Para evaluar las respuestas del chatbot se analizó las variables sexo del usuario y dudas esclarecidas dando por resultado 0.497 como se ve en la Tabla 3 por lo cual es aceptado, lo que quiere decir, que tanto para varones y mujeres sus dudas fueron absueltas por el servicio de consejería.

Tabla 3

Correlación entre el sexo y las dudas resueltas

\begin{tabular}{|c|c|c|c|c|}
\hline \multirow{7}{*}{$\begin{array}{l}\text { Rho de } \\
\text { Spearman }\end{array}$} & & & Sexo & Dudas Resueltas \\
\hline & \multirow[t]{3}{*}{ Sexo } & Coeficiente de correlación & 1,000 & 0,497 \\
\hline & & Sig.(bilateral) & & 0,005 \\
\hline & & $\mathrm{N}$ : muestra & 30 & 30 \\
\hline & \multirow[t]{3}{*}{ DudasResueltas } & Coeficiente de correlación & 0,497 & 1,000 \\
\hline & & Sig.(bilateral) & 0,005 & \\
\hline & & N: muestra & 30 & 30 \\
\hline
\end{tabular}

Para evaluar la calidad de servicio del chatbot, se analizaron las variables entre el sexo del usuario y la calificación del servicio dando por resultado 0.491 según se observa en la Tabla 4 por lo cual es aceptado, es decir, que para varones y mujeres la calificación es buena en base al servicio brindado.

Tabla 4

Correlación entre el sexo y la calificación

\begin{tabular}{lllrr}
\hline \multirow{2}{*}{$\begin{array}{l}\text { Rho de } \\
\text { Spearman }\end{array}$} & Sexo & Coeficiente de correlación & \multicolumn{1}{c}{ Sexo } & \multicolumn{1}{c}{ Calificación } \\
& & Sig.(bilateral) & & 0,491 \\
& & N: muestra & 30 & 0,006 \\
& \multirow{2}{*}{ Calificación } & Coeficiente de correlación & 0,491 & 30 \\
& & Sig.(bilateral) & 0,006 & 1,000 \\
& & N: muestra & 30 & 30 \\
\hline
\end{tabular}

Para evaluar la posibilidad de recomendación del chatbot por parte de los estudiantes, se analizaron las variables entre el sexo del usuario y la recomendación del servicio de Telenanu. El resultado del coeficiente es 0.367 según la Tabla 5 por lo cual es aceptado, lo que quiere decir, que tanto varones como mujeres concuerdan en recomendar el servicio de consejería en salud sexual y reproductiva de Telenanu. 
Tabla 5

Correlación entre el sexo y las recomendaciones

\begin{tabular}{|c|c|c|c|c|}
\hline \multirow{7}{*}{$\begin{array}{l}\text { Rho de } \\
\text { Spearman }\end{array}$} & & & Sexo & Recomendar Nanu \\
\hline & \multirow[t]{3}{*}{ Sexo } & Coeficiente de correlación & 1,000 & 0,367 \\
\hline & & Sig.(bilateral) & & 0,046 \\
\hline & & $\mathrm{N}$ : muestra & 30 & 30 \\
\hline & \multirow[t]{3}{*}{$\begin{array}{l}\text { recomendarNa- } \\
\text { nu }\end{array}$} & Coeficiente de correlación & 0,367 & 1,000 \\
\hline & & Sig.(bilateral) & 0,046 & \\
\hline & & $\mathrm{N}$ : muestra & 30 & 30 \\
\hline
\end{tabular}

Cuando se tratan las redes sociales, hay mucha diversidad al respecto, pero solo algunas son de mayor demanda y dirigida a cierto público específico lo cual se convierte en lo primero a evaluar, a razón de tener presencia en el público al que se quiera llegar. Por otro lado, si bien el proyecto no contempla un estudio a través de entrevistas más sí de un cuestionario, se desarrolló la técnica Wizard of $\mathrm{Oz}$ la cual permite interactuar con el usuario y analizar cuál es el comportamiento frente al entorno de una conversación real con el usuario, Además, en el estudio ejecutado en el capítulo de resultados se pudo concluir que entre las redes sociales de mayor preferencia figuran Instagram y WhatsApp las cuales representaron el $20 \%$ y el $13 \%$ respectivamente

Si bien el servicio de Catalina chatbot, recibió una buena captación de usuarios ello también dependió de la organización relacionada y la capacidad para integrar el chatbot con 79 plataformas web, aunque se discrepa del hecho de tener la misma capacidad para implementar un chatbot a un sitio web, la diversidad es lo que hace rico y versátil para abarcar nuevo público.

Existen diversas plataformas para desarrollar NL; sin embargo, su uso depende de la evaluación que se realice. En esta investigación, se optó por Azure Healt Bot debido a que cuenta con las normas HIPAA para el tratamiento de la información relacionada al ámbito de la salud, la capacidad de integración con otras herramientas. Por otra parte, se considera conveniente usar la página web de la institución debido al ámbito académico aplicado; no obstante, para Telenanu se determinó una página apartada debido a su ámbito de servicio de consejería en salud sexual y reproductiva. Respecto al chatbot de prevención de enfermedades mentales con el uso de la técnica de eliminación de ruido se considera adecuado ya que permite eliminar los ruidos de las señales originales al captar a través de la voz, lo cual es indicado cuando los chatbot tiene esta funcionalidad, respecto a la técnica de mayúscula /minúscula es un factor considerado para comprender mejorar la comprensión de una palabra que haya sido alternada entre mayúscula y minúscula, por lo cual se integró esta técnica en el proyecto.

\section{Conclusiones}

Con el estudio realizado se logró construir un chatbot que interactúa con el estudiante de manera natural, 
garantizando un medio de acceso gratuito al servicio de consejería en salud sexual y reproductiva para los jóvenes universitarios.

Se ha logrado difundir el servicio de tele consejería de salud sexual y reproductiva en tres redes sociales. Entre estas están Facebook, Instagram y TikTok que recibieron una buena aceptación por parte del público usuario.

Se ha logrado desarrollar tres canales: Microsoft Teams, Telegram y la página web, todo esto debido a la aceptación por parte del público dirigido según la encuesta realizada. Respecto al número de canales a integrar a las soluciones de chatbot, va a depender de la popularidad del canal entre los estudiantes en el momento de realizar el estudio, implementar canales es muy simple, lo que asegura su fácil integración.

Existen muchas plataformas que contemplan un entorno de desarrollo completo con procesamiento de lenguaje natural. La elección de una u otra va a depender de la casuística. En esta investigación, la diferencia la marcó, la capacidad de la plataforma de integrar normas de salud certificadas.

Finalmente, elegir una técnica de reprocesamiento es un factor clave para permitir la interacción natural con el agente conversacional.

\section{Referencias}

Acurio Gamarra, J. C., \& Vergara Rodríguez, J. P. (2020, mayo 11). ¿EsSalud debería continuar con la cobertura de atención al mayor de edad hasta los 28 años, tal como sucede con la pensión de alimentos? $L P$. https://lpderecho.pe/essaludcobertura-atencion-mayor-edad28-anos/

Bonales, G., Pradilla, N., \& Martínez, E. (2021). Chatbot como herramienta comunicativa durante la crisis sanitaria COVID-19 en España. ComHumanitas: revista cientifica de comunicación, 11(3),1-22. https:// doi.org/10.31207/rch.v11i3.270

Camacho, A., Oyarzo Torres, S., Murdock, M., \& Bremen de Mucio. (2020). Seminario web sobre la salud sexual y reproductiva en tiempos de COVID-19-OPS/OMS
| Organización Panamericana de la Salud. https://www.paho.org/es/ noticias/3-4- 2020-seminario-websobre-salud-sexual-reproductivatiempos-covid-19

Carbajo Martín, L. (2020). COVID-19 y oportunidad de transformación digital. Revista Clinica de Medicina de Familia, 13(3), 177-179.

ENDES. (2019). Encuesta Demográfica $y$ de Salud Familiar-Endes. https://www.inei.gob.pe/media/ MenuRecursivo/publicaciones_ digitales/Est/End es2019/

García, D. Y. R. (2007). La Sexualidad en los Adolescentes: Algunas Consideraciones.9.

INEI - Perú: Encuesta Demográfica y de Salud Familiar 2019-Nacional y 
Regional. (2019) https://www.inei. gob.pe/media/MenuRecursivo/ publicaciones_digitales/Est/End es2019/

Justiniano, R. K. (2021, abril 11). 18. Teleconsejería en salud sexual $y$ reproductiva en Perú, durante la pandemia: TeleNanu (Spanish) (2021-05-05). Virtual International Day of the Midwife. https:// vidm.org/event/18-teleconsejeriaen- salud-sexual-y-reproductivaen-peru-durante-la-pandemiatelenanu/

Podrazhanshy, A., Han, M., Zhang, H., \& He, S. (2020). A Chatbotbased Mobile Application to Predict and Early-prevent Human
Mental Illness. ACM. https://doi. org/10.1145/3374135.3385319

Sanjuán, C. (2020, junio). (Des) Información Sexual: Pornografía $Y$ Adolescencia. Shevat, A. (2017). Designing Bots. https:/learning. oreilly.com/library/view/designingbots/9781491974810/

Valero Clavel, D. (2019). Desarrollo de un robot conversacional para redes sociales en el dominio académico [Universidad de Alicante]. https://rua.ua.es/ dspace/bitstream/10045/96992/1/ Desarrollo_de_un_robot_conv ersacional_para_redes_social_ Valero_Clavel_Daniel.pdf

Este trabajo fue desarrollado con el auspicio de la Facultad de Obstetricia y Enfermería, la Facultad de Ingeniería y Arquitectura de la Universidad de San Martín de Porres. 